The Agriculturists 5(1\&2): 44-49 (2007)

ISSN-1729-5211

A Scientific Journal of Krishi Foundation

\title{
Genotype Affected Callus Induction and Plant Regeneration from Dehusked Rice (Oryza sativa L.) Seeds
}

\author{
M. Y. Miah'", M. E. Hoque ${ }^{2}$, M. M. Islam², M. Ahmed ${ }^{2}$ \\ Kazi Noor-E- Alam Jewel ${ }^{3}$ \\ ${ }^{1}$ Depar/ment of Soil Science, BSMRAU, Gazipur-1706, Bangladesh, ${ }^{2}$ Agrotechnology Discipline Khulna \\ University, Khulna, Bangladesh. ${ }^{3}$ British American Tobacco Bangladesh (BATB), \\ New DOHS, Mohakhali, Dhaka,Bangladesh \\ * Corresponding author: E-mai.sanjidasy@yahoo.com
}

Received: 02 September 2007

Accepted: 25 November 2007

\begin{abstract}
Callus induction and plant regeneration ability of the dehusked rice genotypes are important tools for varietal improvement. This investigation presents the comparative callus induction and plant regeneration as obtained from the dehusked seeds of rice cultured on MS (Murashige-Skoog) medium. In the experiment conducted in a Complete Randomized Design under laboratory conditions, dehusked seeds of Ranisalute, Chiniatap, Somudrafena, Chiniguri-2 and Katchra rice cultivars grown in South West Bangladesh were tested on autoclaved and agarified MS nutrient medium with four replications to study their callus induction and subsequent plant regeneration ability. Chiniguri-2 yielded the highest calli and plant regeneration.
\end{abstract}

Keywords: Dehusked rice, callus, MS medium

\section{Introduction}

Rice (Oryza sativa and Oryza glaberrima) is the world's most important food crop. So its importance as staple food emphasizes its improvement undoubtedly. A considerable improvement has been achieved through traditional rice breeding (Sun et al., 1990). Now a days, however, various tissue culture techniques are being applied for varietal development of cereal crops including rice in different countries (Dorosieve, 1996). Among the techniques, anther culture, protoplast fusion, leaf culture, root culture and dehusked grain culture are important to exploit novel rice varieties (Ram and Singh, 1998). Several high yielding rice varieties were developed through the application of anther culture in China (Ying,
1983). But plant regeneration from callus is more successful than anther culture (Guo and Cao, 1982). Moreover, plants regenerated from calli show desirable genetic changes such as disease resistance, salt tolerance, and other physiological properties (Kucherenko et al., 1979). Additionally, calli induced by dehusked rice seeds can be successfully used for plant regeneration. These facts suggest that there is a greater possibility of developing novel rice varieties through the appropriate use of dehusked rice induced calli. However, dehusked rice culture technique is still limited by many factors which influence culture efficiency, such as plant genotype (Li, 1991), the culture methods (Yang and Zohu, 1979), the media (Sun et al., 1990) and the culture conditions (Qu and Chen, 1983). 
In this investigation, the comparative callus induction and plant regeneration ability of Ranisalute, Somudrafena, Chiniguri-2, Chiniatap and Katchra rice cultivars cultured on MS medium were evaluated.

\section{Materials and Methods}

Autoclaved and agarified ( $8 \mathrm{~g} \mathrm{~L} \mathrm{~L}^{-1}$ agar) MS nutrient medium (Murashige and Skoog, 1962) containing all needed inorganic major and minor elements, vitamins, amino acids etc. with a $\mathrm{pH}$ of 5.7 which were used for callus induction and plant regeneration. Ten healthy, sterilized $(70 \%$ ethanol $/ 0.2 \% \mathrm{HgCl}$ ) and dehusked rice seeds of the Ranisalute (C1), Somudrafena (C2), Chiniguri-2 (C3), Chiniatap (C4) and Katchra (C5) rice cultivars were inoculated in a cotton plugged conical flask with $25 \mathrm{ml}$ of the MS medium having four replications. With the same number of replication, plant regeneration medium contained $5 \mathrm{ml}$ of the agarified MS medium in $15 \mathrm{~mL}$ sterilized test tubes. For callus induction, agarified MS medium was supplemented with $30 \mathrm{~g} \mathrm{~L}^{-1}$ sucrose. For plant regeneration, agarified MS medium was supplemented with casein hydrolysate $\left(4 \mathrm{~g} \mathrm{~L}^{-1}\right)$ and sucrose $70 \mathrm{~g} \mathrm{~L}^{-1}$.

After inoculation of seeds, the cultures were kept in dark condition for one week at $26 \pm 1^{\circ} \mathrm{C}$. Then the cultures were transferred under 16 hour's photoperiod at light intensity of 3000 Lux. The temperature maintained through out the culture period was $25 \pm 1^{\circ} \mathrm{C}$. After that, the induced calli were transferred into $15 \mathrm{~mL}$ test tubes with the maintenance of the same culture temperature, photoperiod and light intensity. Within one month, greenish plantlets were emerged from the calli. Well rooted plantlets were transferred to the earthen pot containing an autoclaved mixture of vermiculite and sand (1:1). Prior to transfer, the plantlets were washed several times with distilled water to remove the trace of agar. For calculation, calli emerged from one seed was considered as one and the regenerated plants were counted based on the number of callus producing plantlets and the data on callus induction and plant regeneration were collected at the end of one week and the 4th week of a culture month, respectively.

Statistical analysis was carried out by a computer package MSTAT-C and LSD was used for comparison of the treatment means.

\section{Results and Discussion}

\subsection{Callus induction}

During the culture period, dehusked rice seeds of the test cultivars appreciably produced calli (Table 1 and Fig. 1). Although, calli induction was significant $(p \leq 0.01)$, Chiniguri-2 yielded the maximum calli (39.19\%) which was closely followed by Somudrafena and Khatchra (about $36 \%$ ). The lowest calli $(14.06 \%)$ was observed in Ranisalute. The cultivars Ranisalute and Chiniatap did not differ significantly with respect to callus induction and the frequency of callus induction of these two varieties was remarkably lower than those of the other three cultivars. Similarly, callus induction of Somudrafena, Chinigun-2 and Katchra did not differ significantly having $36.02,39.19$ and $36.18 \%$ callus induction, respectively. These sorts of callus induction suggest that the calli formations were influenced by the respective genotypes tested. As for calli formation, these findings also revealed that there was an inter cultivar variation. These findings are in good agreement with those reported by Chung (1980) and Chen (1982) who clearly stated that calli formation varies among genotypes.

\subsection{Plant Regeneration}

As shown in Table 2 and Fig. 2, calli derived from all the cultivars tested for plant regeneration responded significantly $\left(p \_0.01 \%\right)$ in plant regeneration and ranged from 20 to $66.66 \%$. But the minimum plant regeneration (only $20 \%$ ) was observed from the calli of the cultivar Chainiatap. 
Table 1. Effect of variety on callus induction.

\begin{tabular}{lcc}
\hline Cultivar & Seeds inoculated & Callus induction (\%) \\
\hline C1: Ranisalute & 40 & 14.06 \\
C2: Somudrafena & 40 & 36.02 \\
C3: Chiniguri-2 & 40 & 39.19 \\
C4: Chiniatap & 40 & 16.09 \\
C5. Khatchra & 40 & 36.18 \\
CV\% & - & 5.69 \\
LSD (0.01) & - & 3.43 \\
Level of significance & NS & $*$ \\
\hline
\end{tabular}

*= Significant at $1 \%$ level of probability

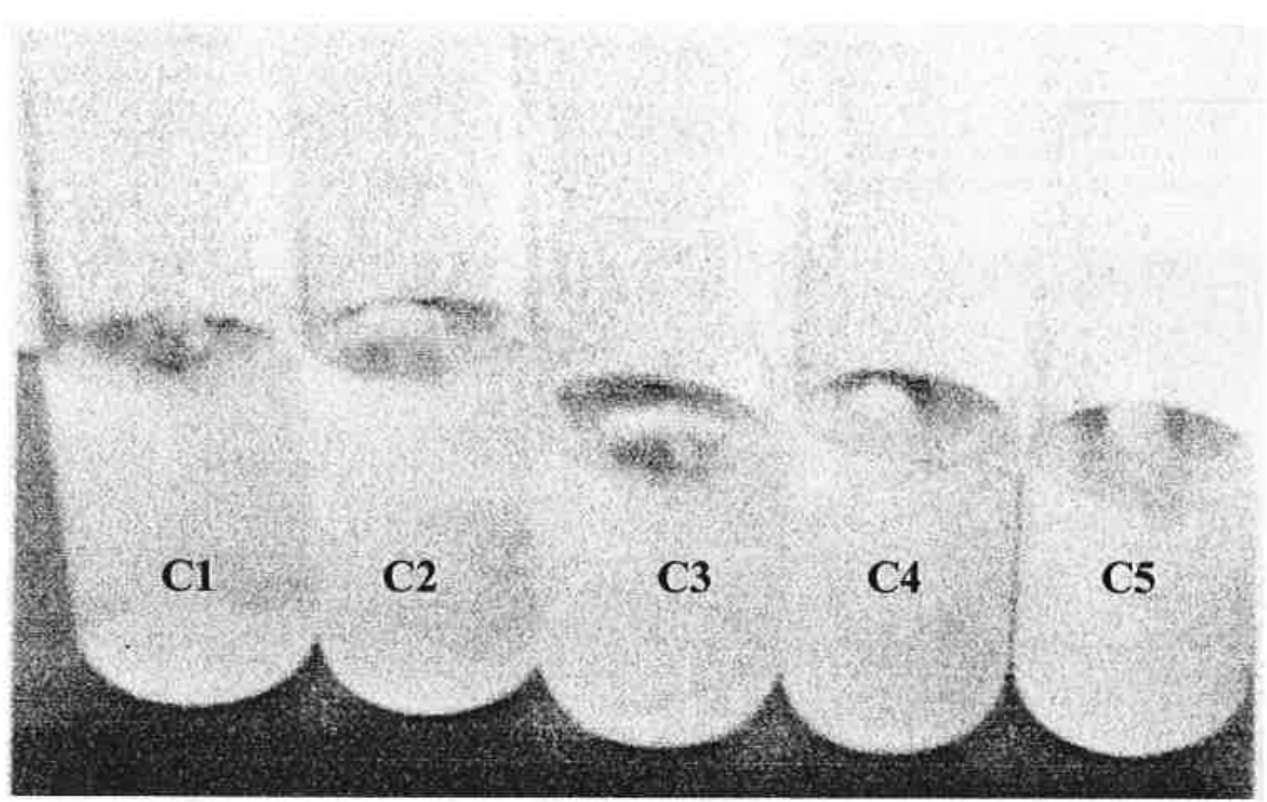

Cl-Ranisalute, C2-Somudrafena, C3-Chiniguri-2, C4-Chinialap , C5-Khatchra

Fig. 1. Callus induction from dehusked rice seeds of the test rice cultivars.

On the contrary, the calli derived from the cultivars Ranisulate, Chiniguri-2 and Khatchra showed comparatively better performance on the plantlets formation $(50,66.66$ and $50 \%$ respectively. However, it is remarkable that Sumodrafena which gave the maximum callus produced only $40 \%$ plantlets. The calli obtained from Chiniguri-2 gave rise to the maximum plant 
regeneration (66.6\%). These sorts of variability in plant regeneration among the cultivars agree well with the findings of Guo and Cao (1982) and Wu and Chen (1987). They confirmed that dehusked rice seeds affected plant regeneration in the different genotypes although genotype to genotype variation was remarkable. The rice cultivars used in the current investigation have shown differential potentiality for both callus induction and plant regeneration. The plants in
Fig. 3 display the well grown regenerated plants derived from the calli of the Chiniguri- 2 cultivar on the autoclaved vermiculite/sand mixture. This remarkable growth of the regenerated plants recognizes the efficacy of the popularly used vermiculite/sand mixture for growing regenerated plants and shows the genotype dependent efficiency of the Chiniguri-2 rice cultivar on callus induction.

Table 2. Plant regeneration from plated calli

\begin{tabular}{lccc}
\hline Cultivar & No. of calli plated & No of plant regenerated & Plant regeneration (\%) \\
\hline C1: Ranisalute & 10 & 5 & 50.00 \\
C2: Somudrafena & 24 & 16 & 40.00 \\
C3: Chiniguri-2 & 20 & 8 & 66.66 \\
C4: Chiniatap & 10 & 2 & 20.00 \\
C5 Khatchra & 12 & 6 & 56.00 \\
CV\% & 27.18 & 14.8 & 6.90 \\
LSD & 1.56 & 2.04 & 4.55 \\
Level of significance & $* *$ & $*$ & $*$ \\
\hline
\end{tabular}

$*=$ Significant at $1 \%$ level of probability

$* *=$ Significant at $5 \%$ level of probability

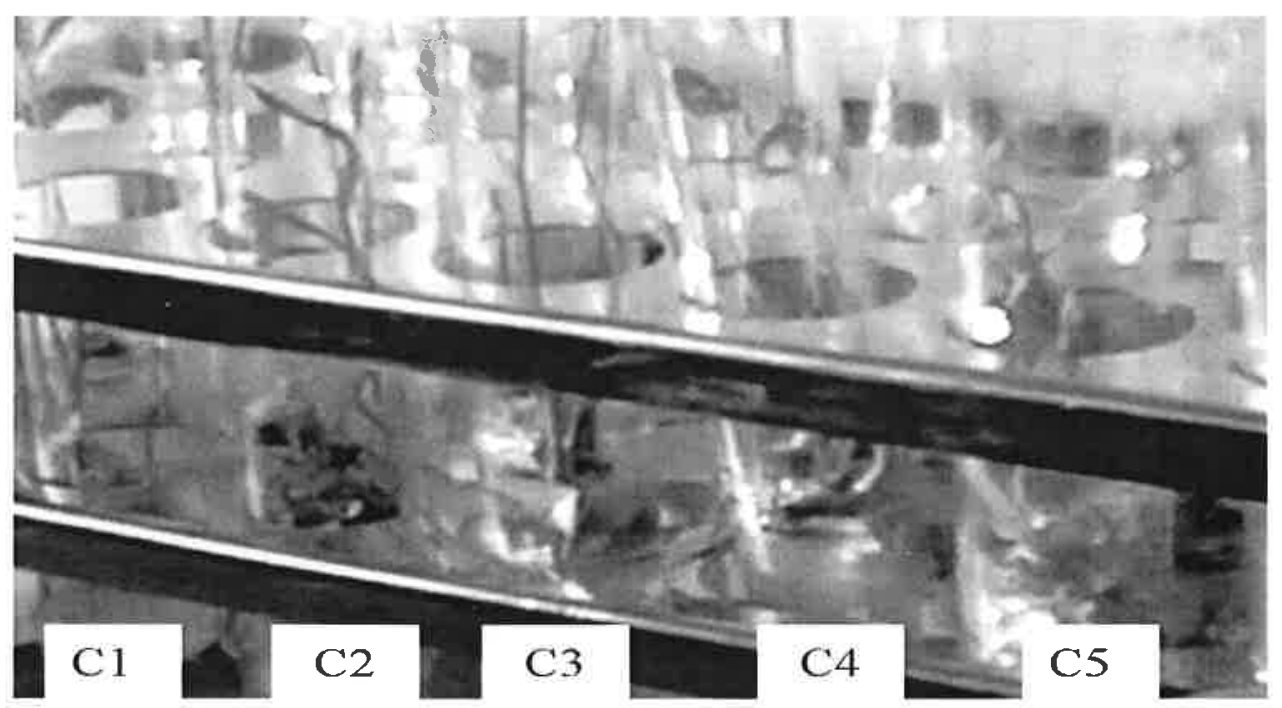

Cultivars: C1-Ranisalute, C2-Somudrafena, C3-Chiniguri-2,C4-Chiniatap, C5-Khatchra

Fig. 2. Regenerated plants from the calli of cultivars 


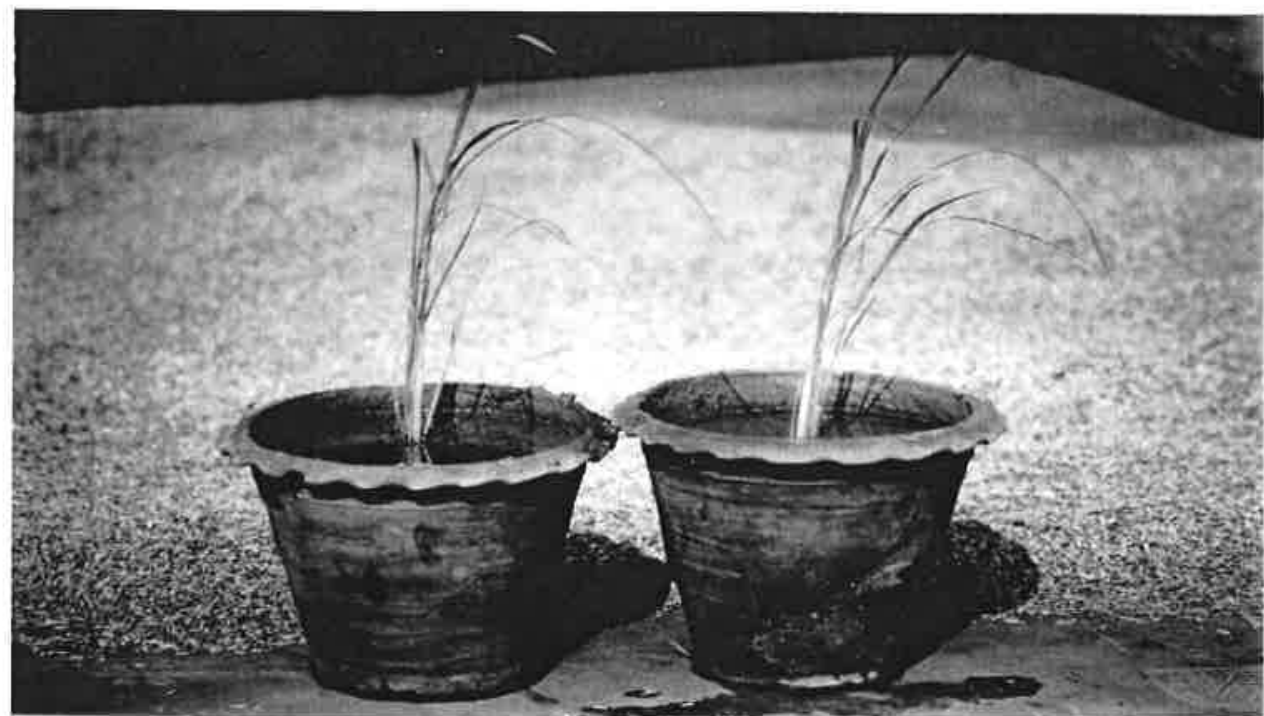

Fig. 3. Rregenerated plants (Chiniguri-2) grown on vermiculite-sand mixture.

\section{Conclusions}

This study revealed that quantitative information on callus induction and plant regeneration could be obtained from the MS medium based cultures of dehusked rice seeds. Additionally, it is evident that callus induction and plant regeneration depended on the genotypes. Further, the cultivars responded well to both callus induction and plant regeneration may be utilized for exploiting somaclonal variant plants as well as in breeding program through tissue culture to reduce the huge requirement of seeds and breeding materials needed for the development of new varieties. However, field study based confirmation on the callus induction and plant regeneration efficiency of the cultivar Chiniguri2 need to be studied.

\section{References}

Chen, J. W. 1982. Study on the factors affecting plantlet regeneration in rice tissue culture. Ziwshenlixue, Torgan, 3: 33-34.
Chung, G. S. 1980. Tissue culture work on rice in Korea. Rice Tissue Planning Conference, IRRI, 28-30 April.

Dorosieve, L. 1996. Plant cell and tissue culturepresent and future prospects. Genetica Selectasia, 19: 356-362.

Guo, C. Y. and Cao Z. Y. 1982. Effects of different genotypes on induction frequency in anther and scutellum culture of maize in vitro. Heredities, 4: 8-10.

Kucherenko, L. A., Kharachenko, P. N. and Davoyan E. I. 1979. Possible use of isolated organ and tissue culture methods in rice broeding. Taknev. I Kletoch. Kultury v. Selektsii , Moscow, USSR, 31$38 \mathrm{pp}$.

Li, M. F. 1991. Breeding of rice, In: Tissue Culture of Field crops, (Ed), C. J. Yan Shanghai, 135- $152 \mathrm{pp}$.

Murashige, T. and F. Skoog. 1962. A revised medium for rapid growth and bioassays with tobacco tissue cultures. Physiologia Plantarum, 15: 497. 
Qu, R. D. and Y. Chen. 1983. A preliminary research on the function of enhancement of callus induction frequency. Acta Phytophysica Sinica, 9: 375-381.

Ram, H. H. and H. G. Sing. 1998. Crop Breeding and Genetics. Kalyani publishers, New Delhi, 90-92 pp.

Sun, Z. R., P. C. Ni, and Z. Z. Hung. 1990. Studies on the analysis of variance and major/minor factors of medium components influencing the efficiency of callus production ability. Acta Argonomica Sinica, 16: 123-130.
Wu, C. Y. and Y. Chen. 1987. Study of the differences between genotypes in anther culture of Japonica rice. Acta Genetica Sinica, 14: 168-174.

Yang, H. Y. and C. Zohu. 1979. Experimental research on the two patays of pollen development (Oryza sativa. L.), Acta Botanica Sinica, 21: 345-351.

Ying, C. 1983. Anther and pollen culture of rice in China. Proceeding of Workshop on Cell and Tissue Culture Technique for Cereal Crop Improvement. Science Press, Beijing, 27-46 pp. 